\title{
Fashion and out of fashion: appearance and disappearance of a novel nest building innovation
}

\author{
Anders P. Møller
}

\begin{abstract}
Background: Nests are composed of and built with different materials that are handled in specific ways. These materials must initially have been used de novo before commonly being incorporated into nests. Plastic and plastic bags were invented in the 1950s, and they are widely distributed in the environment. Birds started picking up plastic from plastic used to cover farm produce such as silage, potatoes, beets and other crops for use in their nests in the 1960s.

Methods: I recorded the frequency of such plastic use by the Blackbird (Turdus merula) in nests in Denmark, starting in 1966, followed by a peak in use in the 1970s and a subsequent decline.

Results: Nests with plastic were initially built earlier in the season than those without plastic, indicating an association between innovation and early reproduction. Plastic use was subsequently selected against because nests with plastic suffered from higher rates of predation than nests without plastic, probably because nests with plastic were easier to locate. However, the elevated risk of nest predation only applied to outdoor nests, but not to nests inside buildings, probably because visually searching nest predators such as corvids do not enter buildings.

Conclusions: These findings show that the dynamics of nest material use depend on the benefits of innovation and the fitness costs of nest predation.
\end{abstract}

Keywords: Fashion, Indoor breeding, Innovation, Nest predation, Turdus merula

\section{Background}

Animals use a high diversity of materials for nest building ranging from branches, twigs, leaves and roots to moss, lichens, seeds, flowers and material of animal origin such as spider silk, feathers, hair and saliva (Collias and Collias 1984; Hansell 2000, 2007). Each type of nest material is handled in a specific way before being incorporated into nests which requires specific behavioral adaptations. How has this immense diversity evolved? When novel material appears, it may fortuitously be incorporated. Because choice of nest material and the way in which it is incorporated is partly innate (Collias and Collias 1984; Hansell 2000, 2007), this implies a genetic component. If not selected against, use of novel nest material

*Correspondence: anders.moller@u-psud.fr

Ecologie Systématique Evolution, Université Paris-Sud, CNRS

AgroParisTech, Université Paris-Saclay, 91405 Orsay Cedex, France will increase in frequency. Indeed, exposure of birds to a number of novel materials only recently produced by humans and subsequently used for nest construction such as paper, plastic and wire has resulted in their incorporation in nests. For example, Magpies (Pica pica) are known to incorporate wires and cloth instead of twigs and roots (Zheng 1962, 1995). Likewise, Chinese Bulbuls (Pycnonotus sinensis) include plastic, paper, cloth, nylon particles and threads of human origin into their nests (Chen et al. 2006; Wang et al. 2009). Such materials are incorporated into nests in proportion to their abundance in the urban environment implying that use reflects availability (Wang et al. 2009). This observation demands the question what limits the innovation and the frequency of use of such novel nest building materials.

Some nest materials have only been used recently simply because they did not exist just a few decades ago. A case in point is plastic used for covering farm produce 
that was not common in the environment until the mid 1960s when the first Blackbird (Turdus merula) nests with plastic pieces were recorded in different parts of Europe (Cramp and Perrins 1988; Glutz von Blotzheim and Bauer 1988; Stephan 1999; this study). The use of novel nest materials may be akin to innovations that attract the attention of conspecifics. Indeed use of feathers by Rock Sparrow (Petronia petronia) females benefitted the nest owners in terms of differential parental investment by the partner (Griggio et al. 2015). Such novel nest materials may resemble traditional material like large dry leaves that are commonly incorporated into nests of Blackbirds (Stephan 1999). Birds' nests are in most cases well camouflaged (Collias and Collias 1984; Hansell 2000, 2007), making any use of conspicuous nest material selected against by nest predators that readily locate visually conspicuous nests. However, predation is often spatially heterogeneous allowing for differential emergence of nest building traditions in the local absence of predators.

The objective of this study was to describe the temporal pattern of emergence and subsequent disappearance of a novel nest building behavior, the use of pieces of plastic in Blackbird nests. The most common nest materials are dried leaves, stalks, roots and earth that account for most nest material. Since plastic is not a limiting resource in human-modified habitats, what limits its use in nests? Here I describe the appearance, increase and eventual demise of plastic use in birds' nests linked to the fitness costs of plastic use. I did that by investigating the link between plastic use and timing of breeding, assuming that this nest building innovation increased in frequency through use by the earliest breeding individuals. Furthermore, I recorded nest predation rates indoors in the absence of most nest predators and outdoors in their presence. Because nest predation is much less common indoors than outdoors (Møller 2010), there should be much weaker selection against plastic use among individuals breeding indoors than outdoors. I investigated these predictions in a long-term study of Blackbirds during 1966-2014. The Blackbird is a very common passerine throughout its range in the Palearctic, where it breeds in a wide range of habitats with particularly high population densities in urban habitats and other habitats modified by humans (Møller et al. 2014). It produces up to four successful broods per year in my study area. Nests are located in bushes and trees and near humans also on or within buildings. Nests are only rarely located on the ground. Only females build nests and incubate, while both sexes provision the offspring (Snow 1958; Cramp and Simmons 1980; Glutz von Blotzheim and Bauer 1988; Stephan 1999). Incubation lasts 12.6 days and the nestling period 13.6 days (Cramp and Simmons 1980).

\section{Methods}

\section{Study site}

The study was conducted at Kraghede $\left(57^{\circ} 12^{\prime} \mathrm{N}, 10^{\circ} 00^{\prime} \mathrm{E}\right)$, Denmark, in an open agricultural habitat with scattered plantations, gardens and hedgerows where Blackbirds are commonly breeding (Møller 1991). In addition, Blackbirds breed commonly inside barns and stables of farms, where they are particularly successful (Møller 2010). The study area comprised $12 \mathrm{~km}^{2}$, but $45 \mathrm{~km}^{2}$ since 1987 .

\section{Data set}

Møller (1991) recorded 169 nests during 1971-1974 and 1979-1982, while Møller (2010) reported a total of 503 nests. Here I report an additional 42 nests recorded during 1966-1971 and 355 nests during 2008-2014. In total this amounted to 643 nests with 132 nests indoor of which 7 were depredated, while 511 nests were located outdoors with 196 depredated.

\section{Egg laying date, nest composition and nest fate}

I searched systematically for Blackbird nests in all suitable habitats (Møller 1991, 2010), and all nests were scored with respect to plastic use with each nest either being scored as having plastic or not.

All nests with eggs were visited once a week, while nests with nestlings were visited when ca. 10 days old and again after the estimated date of fledging. I estimated date of laying of the first egg from incomplete clutches at the first visit, assuming that one egg is laid daily (Snow 1958; Cramp and Simmons 1980; Glutz von Blotzheim and Bauer 1988; Stephan 1999), or from the estimated age of nestlings. Nests containing nestlings at the last visit and an intact empty nest on the following visit were scored as successful, while empty nests that could not have produced fledglings, or were destroyed, were considered as having suffered from predation.

\section{Statistical analyses}

I used logistic regression to test whether the use of plastic as nest material depended on year and year squared (to test for linear and quadratic temporal trends in use), location (indoors or outdoors) and the interaction between nest location and year and year squared, respectively. The interactions were included to test whether the temporal effects depended on nest location. In a GLM with normally distributed errors and an identity link function I assessed whether laying date differed between nests with and without plastic while including nest location (indoors or outdoors) in the statistical model. In a final logistic regression model with nesting success a binomial variable I tested whether nesting success depended on plastic use, nest location (indoors or outdoors) and their interaction to account for the possibility that plastic use was costly 
outdoors but not indoors. The correlations between variables were all weak with the strongest correlation being 0.38 . Hence there were no problems of multi-collinearity. All analyses were made with JMP (SAS 2012).

\section{Results}

I recorded the first case of a Blackbird with plastic in its nest in 1966 followed by a rapid increase first among nests located outdoors and subsequently in nests located indoors (Fig. 1). Nests with plastic almost disappeared after 20 years with only a few observations having been recorded indoors in recent year (Fig. 1). This result was supported by a logistic regression showing significant differences in linear and quadratic terms of year, nest location (indoors or outdoors), and the interaction between location and year and year squared (Table 1). This model fitted the data (likelihood ratio $x^{2}=26.04, \mathrm{df}=61$, $p=0.79$ ), accounting for $27 \%$ of the variance. The highest variance inflation factor was 4 , and hence there was no problems of multi-collinearity.

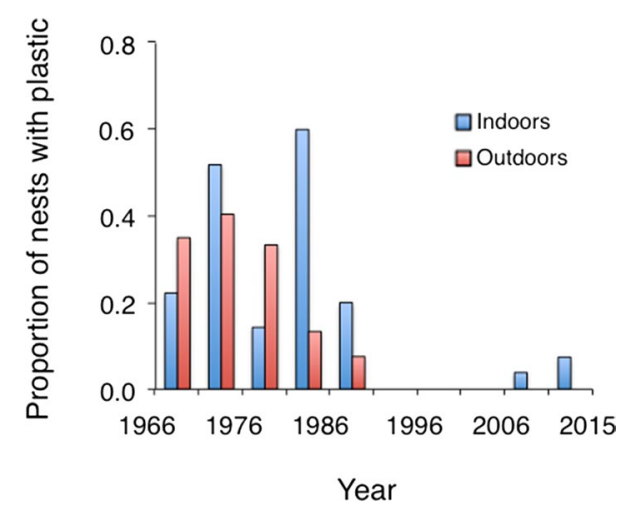

Fig. 1 Proportion of Blackbird nests with plastic located indoors and outdoors

Table 1 Use of plastic in Blackbird nests in relation to nest location (indoors or outdoors), year, year squared and the interactions between year and year squared, respectively, and nest location

\begin{tabular}{lrrrrr}
\hline Term & $\mathbf{X}^{\mathbf{2}}$ & $\mathbf{d f}$ & $\boldsymbol{p}$ & Estimate & \multicolumn{1}{c}{ SE } \\
\hline Intercept & 20.72 & & $<0.0001$ & -518.36 & 113.87 \\
Location & 31.22 & 1 & $<0.0001$ & 1.82 & 0.38 \\
Year & 97.74 & 1 & $<0.0001$ & 0.26 & 0.06 \\
Year * year & 19.84 & 1 & $<0.0001$ & 0.008 & 0.002 \\
Location * year & 24.93 & 1 & $<0.0001$ & 0.20 & 0.006 \\
Location * year * year & 7.78 & 1 & 0.0053 & 0.006 & 0.002 \\
\hline
\end{tabular}

The logistic regression model had the likelihood ratio $x^{2}=254.85, \mathrm{df}=5$, $p<0.0001$
Plastic use was primarily by Blackbirds breeding early in the season with a significant difference between birds with and without plastic (Table 2), suggesting that early breeding birds were responsible for this novel innovation. In contrast, there was no significant difference between indoor and outdoor nests, while the interaction between plastic use and nest location was significant (Table 2), implying that laying date was earlier for nests with plastic among outdoor nests (mean $\pm \mathrm{SE}=40.3 \pm 1.4$ ) than indoor nests $(42.8 \pm 1.7)$, while for nests without plastic the difference between outdoor and indoor nests was larger (Table 2, the significant location by plastic interaction: no plastic, outdoor: $66.7 \pm 0.7$, no plastic, indoor: $59.3 \pm 1.6)$. The highest variance inflation factor was 1.2 , and hence there was no problems of multi-collinearity.

Plastic use was related to the risk of predation (likelihood ratio $\chi^{2}=6.73, \mathrm{df}=1, p=0.0002$ ). While $29.1 \%$ of 556 nests without plastic were depredated, $49.4 \%$ of 87 nests with plastic were depredated.

Predation, nest location and the interaction between plastic and nest location were all significant predictors of nest success (Table 3). This model fitted the data (likelihood ratio $\chi^{2}=643.00, \mathrm{df}=639, p=0.45$ ). Nests with plastic were less often successful than nests without plastic (Fig. 2; Table 3). Nests indoors were more often successful than nests outdoors (Fig. 2; Table 3). Finally, there was a significant interaction between plastic use and nest location with outdoor nests suffering more from nest predation in the presence of plastic than indoor nests,

Table 2 Laying date ( $1=$ May 1 st) of Blackbirds in relation to plastic use in nests, nest location (indoors or outdoors), and their interaction

\begin{tabular}{lrrrrr}
\hline Term & $\mathbf{X}^{\mathbf{2}}$ & $\mathbf{d f}$ & $\boldsymbol{p}$ & Estimate & \multicolumn{1}{c}{$\mathrm{SE}$} \\
\hline Intercept & 1222.45 & & $<0.0001$ & 52.282 & 0.864 \\
Plastic & 138.53 & 1 & $<0.0001$ & -10.743 & 0.864 \\
Location & 1.94 & 1 & 0.16 & -1.206 & 0.864 \\
Plastic * location & 8.22 & 1 & 0.0041 & 2.485 & 0.864 \\
\hline
\end{tabular}

The GLM model had the likelihood ratio $\mathrm{X}^{2}=209.91, \mathrm{df}=3, p<0.0001$

Table 3 Success of Blackbird nests in relation to plastic use in nests, nest location (indoors or outdoors), and their interaction

\begin{tabular}{lrccrl}
\hline Term & $\mathbf{X}^{\mathbf{2}}$ & df & $\boldsymbol{p}$ & Estimate & SE \\
\hline Intercept & 46.24 & & $<0.0001$ & -1.248 & 0.226 \\
Plastic & 3.12 & 1 & 0.08 & 0.451 & 0.226 \\
Location & 81.43 & 1 & $<0.0001$ & -1.493 & 0.226 \\
Plastic * location & 4.57 & 1 & 0.033 & 0.451 & 0.226 \\
\hline
\end{tabular}

The logistic regression model had the likelihood ratio $x^{2}=98.31$, $\mathrm{df}=3$, $p<0.0001$ 


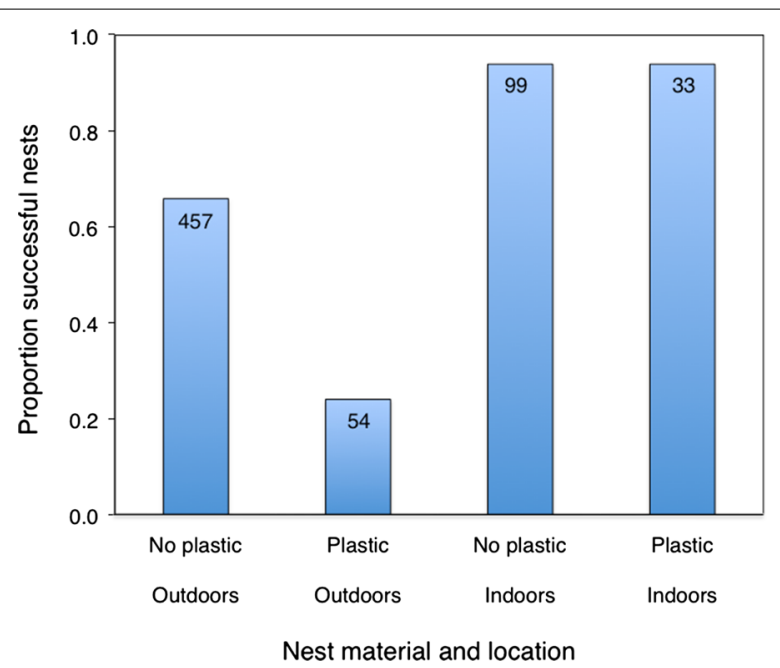

Fig. 2 Proportion of successful Blackbird nests in relation to presence or absence of plastic and whether nests were located indoors or outdoors. Numbers refer to the total number of nests for each category

while predation risk did not differ between indoor nests with or without plastic (Fig. 2; Table 3). The highest variance inflation factor was 1.2 , and hence there was no problems of multi-collinearity.

\section{Discussion}

Blackbirds displayed a novel innovation by using plastic as a nest material starting in 1966. The frequency of this behavior changed over time differing between nests located indoors and outdoors. The first birds starting this innovation were early breeders. There was a fitness cost to plastic use because nests with plastic were more likely to be depredated than nests without plastic, although this pattern was only prominent in nests located outdoors. These observations have implications for our knowledge of behavioral innovations and the spread of traditions.

The use of plastic as a nest material can be considered a novel fashion since the frequency of use started in early breeders, which are known to recruit disproportionately many offspring (Snow 1958). Such innovative individuals can be considered 'trend-setters' by initiating plastic use and through social learning transmit this behavior to conspecifics. There are few examples of the emergence of innovations and the identity of the individuals responsible for such novel behavior. An exception is the emergence of a tradition of washing sweet potatoes by Japanese Macaques (Macaca fuscata) that allowed removal of sand from food items (Kawamura 1959), where the initial behavior emerged in a specific old female. Another example concerns the opening of milk bottles by birds, in particular Great Tits (Parus major), and the consumption of cream, although in that case the innovator was not known (Fisher and Hinde 1949).

Although behavioral innovations are common, only a few novel behavioral patterns spread (Kummer and Goodall 1985). The dynamics of innovations will depend on benefits and costs of such novel behavior, but also on stochastic events that may cause the loss of innovations. Although novel behavior has been described previously, there are few examples. The present study suggested that plastic use in nests was a signal of innovation ability because early breeding females showed a higher frequency of plastic use than later birds. This explanation may also apply to feather use in Rock Sparrows, which is linked to sexual selection for female innovation by choosy males (Griggio et al. 2015). Other studies have also emphasized the role of innovation as a component of sexual selection (Miller 2000; Simonton 2003). I have previously shown that nest predation is a strong selective agent for birds breeding in association with humans and human habitation (Møller 2010). Two species that have just started this association such as Song Thrush (Turdus philomelos) and Chaffinch (Fringilla coelebs) have a low frequency of indoor nests, while species that have been associated with humans for millennia such as House Sparrows (Passer domesticus) and Barn Swallows (Hirundo rustica) have a high frequency of breeding in the proximity of humans (Møller 2010). The subsequent dynamics of plastic use showed a peak during the $1970 \mathrm{~s}$ followed by a decline that differed between indoor and outdoor nests. The present study suggests that plastic may have been retained as a nest material in nests located indoors. The disappearance of this behavior was linked to fitness costs of nest predation. There was little or no evidence of plastic being a limiting resource, and most farms with Blackbirds breeding indoors still have ample supply of plastic that is rarely used as a nest material. The fraction of the Blackbird population that breeds indoors is minute compared to the outdoor population in 'natural' habitats. Therefore, the use of plastic first disappeared outdoors and then eventually indoors, as the small fraction that constitutes the indoor population was swamped by Blackbirds from the outdoor population.

\section{Conclusion}

I have shown that a novel behavior (plastic use in nests of Blackbirds) spread rapidly by starting out in early breeders. Fitness costs of this innovation were differentially related to the frequency of outdoor nests apparently because of higher predation risk outdoors due to visually searching nest predators. These findings have implications for our knowledge of the spread of innovations. 


\section{Acknowledgements}

T. Duch provided early encouragement for recording birds' nests and their contents.

\section{Competing interests}

The author declares that he has no competing interests.

Received: 24 April 2017 Accepted: 12 June 2017

Published online: 29 June 2017

\section{References}

Chen W, Guo Z, Hu JC, Yu ZW. Breeding habitat growth and development of Pycnonotus sinensis in Nanchong, China. Chin J Zool. 2006;41:107-11.

Cramp S, Perrins CM. The birds of the Western Palearctic, vol. 5. Oxford: Oxford University Press; 1988

Cramp S, Simmons KEL. The birds of the Western Palaearctic, vol. 8. Oxford: Oxford University Press; 1980.

Collias NE, Collias EC. Nest building and bird behavior. Princeton: Princeton University Press; 1984

Fisher J, Hinde RA. The opening of milk bottles by birds. Brit Birds. 1949;42:347-57.

Glutz von Blotzheim UN, Bauer KM. Handbuch der Vögel Mitteleuropas, vol. 11. Wiesbaden: AULA-Verlag; 1988.

Griggio M, Valera F, Casa A, Pilastro A. Males prefer ornamented females: a field experiment of male choice in the rock sparrow. Anim Behav. 2015;69:1243-50.

Hansell MH. Bird nests and construction behaviour. Cambridge: Cambridge University Press; 2000.
Hansell MH. Built by animals. Oxford: Oxford University Press; 2007.

Kawamura S. The process of sub-culture propagation among Japanese macaques. Primates. 1959;2:43-60.

Kummer H, Goodall J. Conditions of innovative behaviour in primates. Phil Trans R Soc Lond B. 1985:308:203-14.

Miller GE. The mating mind. New York: Doubleday; 2000.

Møller AP. Clutch size, nest predation and distribution of avian unequal competitors in a patchy environment. Ecology. 1991;72:1336-49.

Møller AP. The fitness benefit of association with humans: elevated success of birds breeding indoors. Behav Ecol. 2010;21:913-8.

Møller AP, Jokimäki J, Skorka P, Tryjanowski P. Loss of migration and urbanization in birds: a case study of the blackbird (Turdus merula). Oecologia. 2014;175:1019-27.

SAS. JMP version 10.0. Cary: SAS Inc.; 2012.

Simonton DK. Human creativity: two Darwinian analyses. In: Reader SM, Laland KN, editors. Animal innovation. Oxford: Oxford University Press; 2003. p. 309-25.

Snow DW. A study of blackbirds. Tring: British Museum of Natural History; 1958

Stephan B. Die Amsel. Wittenberg-Lutherstadt: Neue Brehm-Bücherei; 1999.

Wang Y, Chen S, Blair RB, Jiang P, Ding P. Nest composition adjustments by Chinese Bulbuls Pycnonotus sinensis in an urbanized landscape of Hangzhou (E China). Acta Ornithol. 2009;44:185-92.

Zheng GM. The nest building habits of birds. Bull Biol. 1962;2:18-21 (in Chinese).

Zheng GM. Ornithology. Beijing: Beijing Normal University Publishing House; 1995 (in Chinese)

\section{Submit your next manuscript to BioMed Central and we will help you at every step:}

- We accept pre-submission inquiries

- Our selector tool helps you to find the most relevant journal

- We provide round the clock customer support

- Convenient online submission

- Thorough peer review

- Inclusion in PubMed and all major indexing services

- Maximum visibility for your research

Submit your manuscript at www.biomedcentral com/submit 